\title{
NUCLEAR SPIN RELAXATION IN PERIODICALLY PERTURBED SYSTEMS. II. LIKE AND UNLIKE SPINS*
}

\author{
J.S. BLICharsKi aNd A. Wolak \\ Institute of Physics, Jagiellonian University, Reymonta 4, 30-059 Kraków, Poland
}

(Received June 15, 1992)

The effective relaxation time $T_{2 \mathrm{e}}$ is calculated in the weak collision case for a system consisting of resonant nuclear spins $I$ and nonresonant spins $S$, perturbed by periodic sequences of r.f. pulses and dipole-dipole interactions.

PACS numbers: $76.60 .-\mathrm{k}$

The aim of this paper is to calculate effective relaxation time $T_{2 \mathrm{e}}$ for heteronuclear spin system in the presence of r.f. multipulse sequences of arbitrary phase and nutation angles. This theory may be applied for heterogeneous systems with slow molecular motion.

Dipolar interaction between resonant nuclear spins $I-I$ (like spins) as well as heteronuclear dipolar interactions $I-S$ between resonant and nonresonant spins (unlike spins) have been taken into account. A weak collision approximation has been applied. All calculations have been done in the way described in the previous paper (Part I of the series [7]) for homonuclear dipolar interactions. The results presented below are valid also in the case of the dipolar interactions $I-S$ between nuclear spins $I$ and electron spins $S$ (e.g. paramagnetic ions).

Let us consider generalized OWMW pulse sequence [1-7]

$(\pi / 2)_{y}-\left[-\tau-\beta_{x}-2 \tau-\beta_{x}-\cdots-2 \tau-\beta_{x}-\tau\right]_{n}$,

where $\beta_{x}=2 \pi P / N$ is the nutation angle of the very short (delta) r.f. pulse, $T_{\mathrm{c}}=2 N \tau$ is the repetition period of the cycle, $\omega_{\mathrm{c}}=2 \pi / T_{\mathrm{c}}$ is the cycle frequency, $P, N, n$ are natural numbers.

In the calculation we have taken into account secular and nonsecular terms of the dipolar interactions. Using the operator formalism presented in [7] and assuming $\omega_{S} \gg \omega_{\mathrm{c}}, \omega_{I} \gg \omega_{\mathrm{c}}$, one can calculate the effective relaxation time $T_{2 \mathrm{e}}$ as a sum of relaxation contributions from homo- and heteronuclear dipolar interactions $I-I$ and $I-S$, respectively:

$$
\frac{1}{T_{2 \mathrm{e}}}=\left(\frac{1}{T_{2 \mathrm{e}}}\right)_{I I}+\left(\frac{1}{T_{2 \mathrm{e}}}\right)_{I S}
$$

*This work was supported by the Research Grant KBN 224119102 (Poland). 


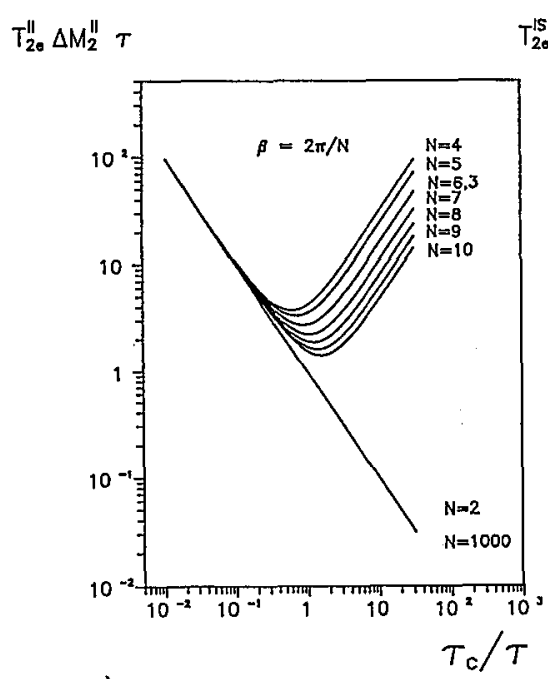

a)
$T_{2 \mathrm{o}}^{\mathrm{S}} \Delta \mathrm{M}_{2}^{\mathrm{IS}} \tau$

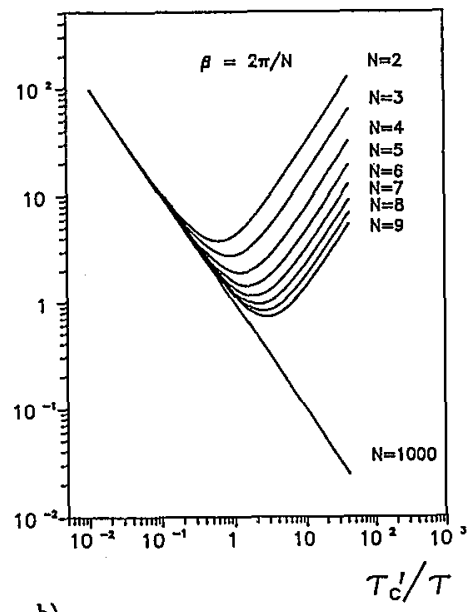

b)

Fig. 1. Effective spin-spin relaxation time $T_{2 \mathrm{e}}$ as a function of $\tau_{\mathrm{c}} / \tau\left(\tau_{\mathrm{c}}^{\prime} / \tau\right)$ for: (a) dipolar interactions between like spins $(I-I)$, (b) dipolar interactions betwcen unlike spins $(I-S)$.

$$
\begin{gathered}
\left(\frac{1}{T_{2 \mathrm{e}}}\right)_{I I}=\left(\frac{1}{T_{2}}\right)_{I I}-\Delta M_{2}^{I I} \tau \frac{\tanh u}{u^{2}} \frac{\sin ^{2} \beta}{1-(\cos \beta / \cosh u)^{2}} \\
\left(\frac{1}{T_{2 \mathrm{e}}}\right)_{I S}=\left(\frac{1}{T_{2}}\right)_{I S}-\Delta M_{2}^{I S} \tau \frac{\tanh (2 w)}{2 w^{2}} \frac{1-\cos \beta}{1-\cos \beta / \cosh 2 w} \\
\left(\frac{1}{T_{2}}\right)_{I I}=\frac{\Delta M_{2}^{I I}}{3}\left(3 \tau_{\mathrm{c}}+\frac{5 \tau_{\mathrm{c}}}{1+\omega_{I}^{2} \tau_{\mathrm{c}}^{2}}+\frac{2 \tau_{\mathrm{c}}}{1+4 \omega_{I}^{2} \tau_{\mathrm{c}}^{2}}\right) \\
\left(\frac{1}{T_{2}}\right)_{I S}=\frac{1}{4} \Delta M_{2}^{I S} \tau_{\mathrm{c}}^{\prime} \quad\left(4+\frac{3}{1+\omega_{I}^{2} \tau_{c}^{\prime 2}}+\frac{1}{1+\left(\omega_{S}-\omega_{I}\right)^{2} \tau_{c}^{\prime 2}}\right. \\
\left.+\frac{6}{1+\omega_{S}^{2} \tau_{c}^{\prime 2}}+\frac{6}{1+\left(\omega_{S}+\omega_{I}\right)^{2} \tau_{c}^{\prime 2}}\right)
\end{gathered}
$$

where

$$
\begin{aligned}
& \Delta M_{2}^{I I}=\left(M_{2}^{I I}\right)_{\mathrm{RL}}-\left(M_{2}^{I I}\right), \\
& \Delta M_{2}^{I S}=\left(M_{2}^{I S}\right)_{\mathrm{RL}}-\left(M_{2}^{I S}\right), \\
& \frac{1}{\tau_{\mathrm{c}}^{\prime}}=\frac{1}{\tau_{\mathrm{c}}}+\frac{1}{\tau_{\mathrm{s}}}, \quad w=\frac{\tau}{\tau_{\mathrm{c}}^{\prime}}=u+v, \quad u=\frac{\tau}{\tau_{\mathrm{c}}}, \quad v=\frac{\tau}{\tau_{S}},
\end{aligned}
$$

$\tau_{\mathrm{c}}$ is the correlation time of molecular motions, $\tau_{S}$ is the relaxation time for spins $S, \tau_{c}^{\prime}$ is effective correlation time, $\Delta M_{2}^{I I}$ and $\Delta M_{2}^{I S}$ are changes of the second moment of the resonance line in the case of motional narrowing (compared with $M_{2}$ for a rigid lattice) for homo- and heteronuclear interactions, respectively. 


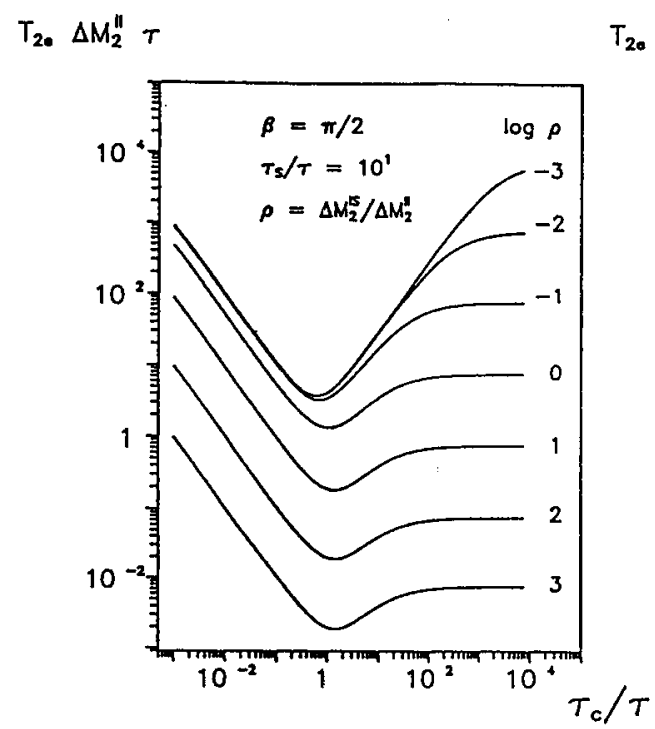

$T_{20} \Delta M_{2}^{n} \tau$

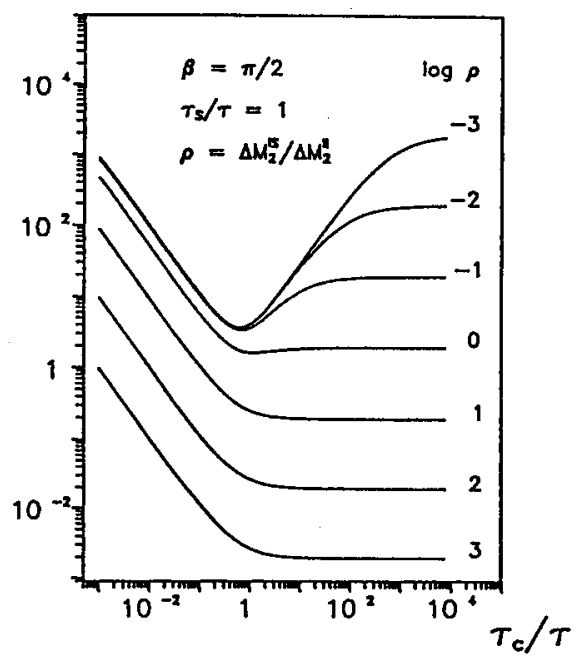

$T_{20} \Delta M_{2}^{n} \tau$

$T_{20} \Delta M_{2}^{\mathrm{R}} T$
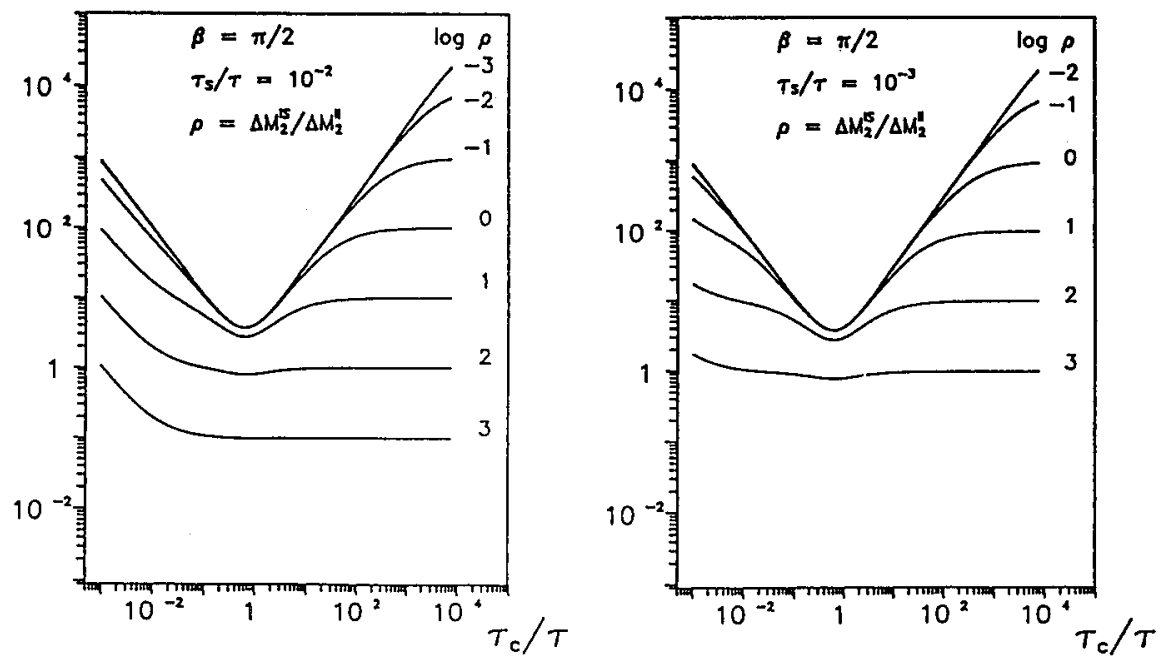

Fig. 2. Effective spin-spin relaxation time $T_{2 \mathrm{e}}$ as a function of correlation time of molecular motions calculated for different values of the parameters $\tau_{S} / \tau$ and $\rho=$ $\Delta M_{2}^{I S} / \Delta M_{2}^{I I}$. Calculations have been done for OWMW pulse sequence $(\beta=\pi / 2)$. Contributions from homo- and heteronuclear dipolar interactions have been taken into account.

Results of numerical calculations of the effective relaxation time $T_{2 \mathrm{e}}$ as a function of correlation time $\tau_{\mathrm{c}}$ are presented in Fig. 1 and Fig. 2. From Fig. 1a, one can see that for $\beta=\pi / 2$ the relaxation time $T_{2 \mathrm{e}}^{I I}$ has a minimum for $\tau_{\mathrm{c}} / \tau=0.62$. If the 

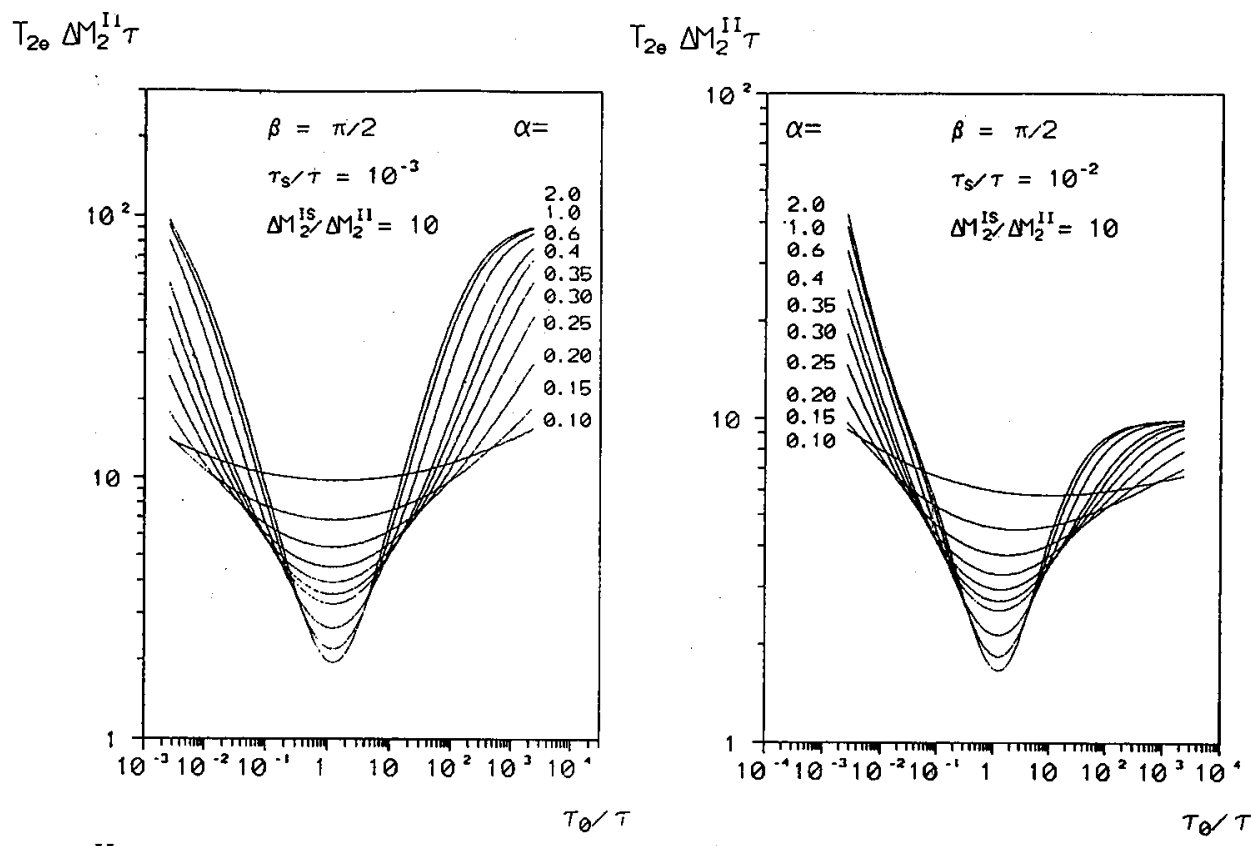

$T_{2 \theta} \Delta M_{2}^{I I} \tau$

$T_{0} T$
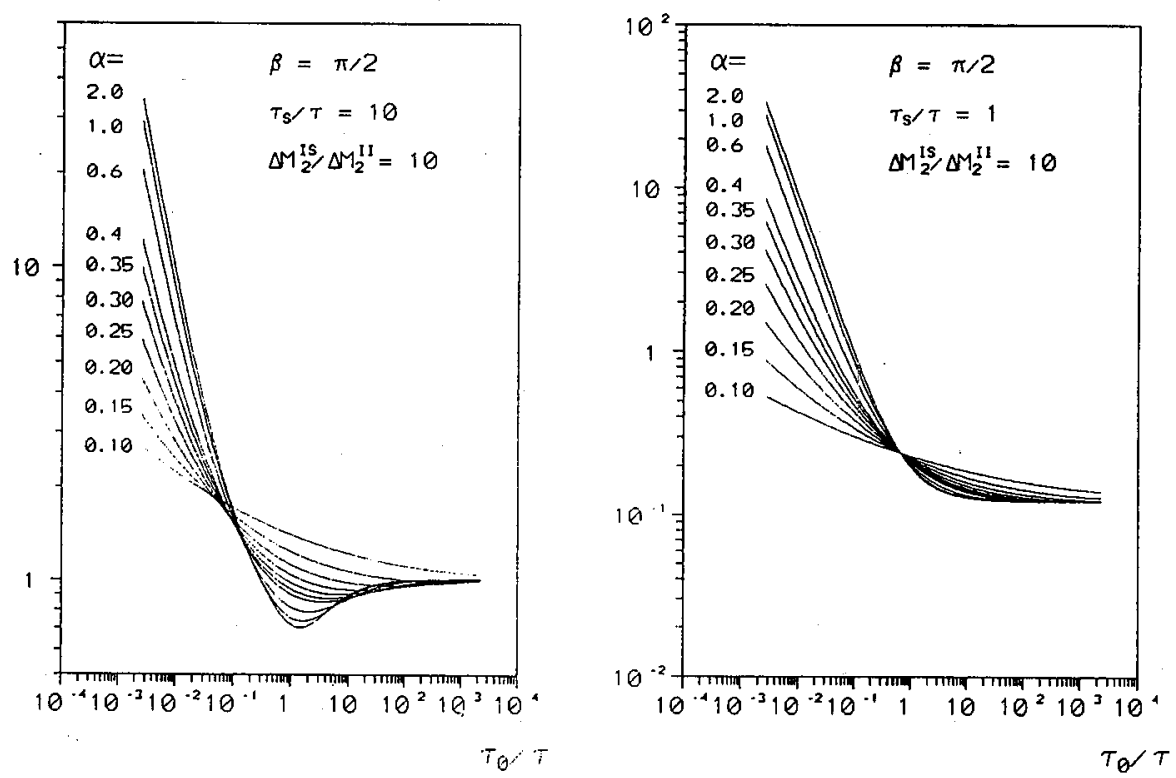

Fig. 3. Effective spin-spin relaxation time $T_{2 \mathrm{e}}$ as a function of $\tau_{0} / \tau$ in the presence of Log-Gauss distribution of correlation time $\tau_{c}$ of the molecular motion. Contributions from homo- and heteronuclear dipolar interactions have been taken into account. Calculations have been done for OWMW pulse sequence $(\beta=\pi / 2)$. 
nutation angle $\beta$ decreases, the value of $\left(T_{2 \mathrm{e}}^{I I}\right)_{\min }$ also decreases and the minimum of $T_{2 \mathrm{e}}^{I I}$ shifts in the direction of longer correlation times (lower temperatures). The relaxation time $T_{2 \mathrm{e}}^{I S}$ exhibits a similar dependence on $\tau_{\mathrm{c}}^{\prime}$ as $T_{2 \mathrm{e}}^{I I}$ (Fig. $1 \mathrm{~b}$ ) but $T_{2 \mathrm{e}}^{I S}$ has a minimum also for Carr-Purcell-Meiboom-Gill pulse sequence $(\beta=\pi)$.

Results of calculation of the resultant relaxation time $T_{2 \mathrm{e}}$ as a function of correlation time of molecular motions are presented in Fig. 2 for $\beta=\pi / 2$ and for different values of the parameters $\tau_{S} / r$ and $\rho=\Delta M_{2}^{I S} / \Delta M_{2}^{I I}$. With increasing $\rho$, the contribution from the heteronuclear interaction increases. For large values of $\rho$ and $\tau \ll \tau_{S}=$ const., the temperature dependence of $T_{2 \mathrm{e}}$ is very weak. As $\rho$ increases, the values of $T_{2 \mathrm{e}}$ decreases. This effect is especially visible for $\tau_{S} \leq \tau$. For $\tau_{S}=\tau$ we observe shortening of $T_{2 \mathrm{e}}$ as well as flattening of temperature dependence of $T_{2 \mathrm{e}}$ in the region of low temperatures $\left(\tau_{\mathrm{c}}<\tau\right)$.

In the case of heterogeneous systems (e.g. biological systems) one can expect a presence of distribution of the correlation times $\tau_{\mathrm{c}}$ for molecular motions. Assuming Log-Gauss distribution of the correlation times

$$
f(s)=\frac{\alpha}{\sqrt{\pi}} \exp \left(-\alpha^{2} s^{2}\right)
$$

with $s=\ln \tau_{\mathrm{c}} / \tau$ and fast exchange (or fast spin diffusion) one can calculate $T_{2 \mathrm{e}}$ as a function of the average correlation time $\tau_{0}$ in the logarithmic scale.

The results of numcrical calculations are presented in Fig. 3 for $\rho=\Delta M_{2}^{I S} / \Delta M_{2}^{I I}=10$ at different values of $\tau_{S} / \tau$ and parametcrs $\alpha$.

The above presented theory will be applied for interpretation of the temperature dependence of $T_{2 \mathrm{e}}$ for protons in the photosynthctic membranes.

\section{Acknowledgments}

The author (JSB) wishes to thank Prof. M. Bloom for help ful discussions during a scientific visit at the University of British Columbia in Vancouver, Canada.

\section{References}

[1] E.D. Ostroff, J.S. Waugh, Phys. Rev. Lell. 16, 1097 (1966).

[2] P. Mansficld, D. Ware, Phys. Lell. 22, 133 (1966).

[3] J.S. Waugh, C.H. Wang, Phys. Rev. 162, 209 (1967).

[4] P. Mansfield, D. Ware, Phys. Rev. 168, 318 (1968).

[5] W. -K. Rhim, D.P. Burum, A.D. Elleman, J. Chem. Phys. 68, 692 (1978).

[6] A.J. Vega, R.W. Vaughan, J. Chem. Phys. 68, 1958 (1978).

[7] J.S. Blicharski, A. Wolak, Acla Phys. Pol. A 79, 591 (1991), part I. 